\title{
Magnetorotational mechanism of explosions of core-collapse supernovae
}

\section{G.S.Bisnovatyi-Kogan *}

Space Research Institute, Profsoyuznaya str. 84/32, Moscow 117997, Russia, and National Research Nuclear University "MEPHI", Kashirskoye Shosse, 31, Moscow 115409, Russia

E-mail: gkogandiki.rssi.ru

\section{S.G.Moiseenko}

Space Research Institute, Profsoyuznaya str. 84/32, Moscow 117997, Russia

E-mail: moiseenkodiki.rssi.rul

\section{N.V.Ardeljan}

Department of Computational Mathematics and Cybernetics, Moscow State University,

Vorobjevy Gory, Moscow B-234, Russia

E-mail: ardeLdcs.msu.su

Core-collapse supernovae are accompanied by formation of neutron stars. The gravitation energy is transformed into the energy of the explosion, observed as SN II, SN Ib,c type supernovae. Results of 2-D MHD simulations are presented, where the source of energy is rotation, and magnetic field serves as a "transition belt" for the transformation of the rotation energy into the energy of the explosion. The toroidal part of the magnetic energy grows with time due to differential rotation. When the twisted toroidal component strongly exceeds the poloidal field, magneto-rotational instability develops, leading to a drastic acceleration in the growth of magnetic energy. Finally, a fast MHD shock is formed, producing a supernova explosion. Mildly collimated jet is produced for dipole-like type of the initial field.

XI Multifrequency Behaviour of High Energy Cosmic Sources Workshop

25-30 May 2015

Palermo, Italy

\footnotetext{
* Speaker.
} 


\section{Introduction}

Core-collapse $\mathrm{SNe}$ explode at the end of evolution of massive stars, with initial mass larger than $\sim 12 M_{\odot}$. The evolution proceeds until the formation of the iron core, and the star collapses due to a loss of a hydrodynamic stability. During the core collapse and formation of a neutron star, gravitational energy release $\sim 6 \cdot 10^{53} \mathrm{erg}$, is carried away by neutrino. The first mechanism suggested in [1]] for the explanation of of the explosion in a core-collapse SN was connected with a neutrino deposition. The huge energy flux carried by neutrino heats the infalling outer layers, reverse the direction of motion, and leads to formation of the shock wave, producing explosions of SN II, SN Ib,c types. More accurate calculations revealed that the energy of such explosion is not enough for the explanation of observations. Many modifications of the neutrino model have been calculated during years, but the problem is not yet clear, see [ $[\mathbb{}]$. One of the most promising modification was a discovery of the convective regions in the layers with a strong neutrino flux from the center (neutrino convection) [[ष]]. The convection carries out the inner hot layers, leading to radiation of a more energetic neutrino. The cross-section of neutrino interaction with matter is proportional to the square of the neutrino energy. so neutrino flux heats stronger the outer layers, leading to formation of a stronger shock. 2-D calculations did not give a definite answer about efficiency of the neutrino convection for formation of a strong shock with the ejection energy sufficient for observational value. The hope was that the situation could be improved in 3-D model.

Nevertheless, simple evaluations lead to the opposite conclusions, that 3-D models give a less powerful explosion. It is clear, that a matter coming from deeper layers is hotter, so the convective eddies of a larger size are more efficient in heating of the envelope by neutrino flux. But it is well known from hydrodynamic experiments and numerical simulations, that in 2-dimensional layer the mechanical convective energy flux goes to larger scale eddied, while in a more realistic 3-D case the large scale eddies loose their energy to formation of smaller eddies. Decrease of the size of convective eddies in realistic 3-D problem makes 3-D models less effective for energy release by neutrino deposition.

In recent simulations we have found that for extremely strong initial magnetic field $H_{0}=10^{12} \mathrm{G}$ a prompt supernova explosion occurs, and collimated jet is formed in agreement with [29]. For case when the initial magnetic field is weaker $H_{0}=10^{9} \mathrm{G}$ we have identified, after the linear growth of the poloidal magnetic field due to differential rotation, the exponential field growth due to the magnetorotational instability of Tayler type [ $\mathrm{B}]$ ]. We call the combination of differential rotation with the Tayler type MRI instability, as magneto-differential-rotation instability (MDRI). Note that magnetorotational explosion (MRE) is supported mainly by the magnetic pressure, which is hardly connected with the scale of eddied, so we may expect the same efficiencies of MRE in 2 and 3-D numerical models.

The ejection due to the explosion is only weakly collimated, while [ㅁ] had obtained a strong collimation in this variant also. In these simulations we considered a uniform magnetic field along the rotational axis, as the initial field configuration, similar to [29].

\section{Magnetorotational mechanism of explosion}

In magnetorotational explosion the transformation of the rotational energy of the neutron star 

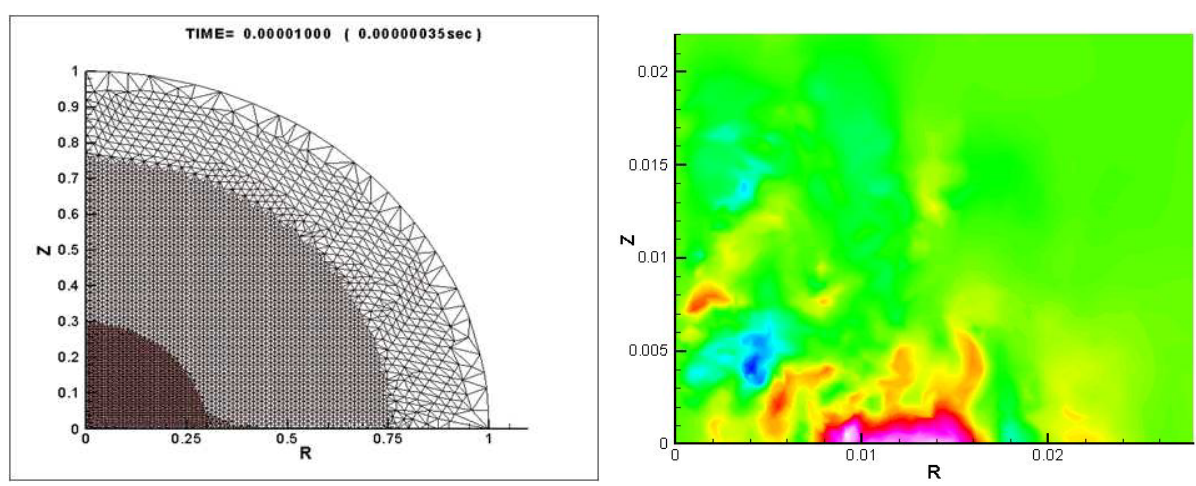

Figure 1: Example of the triangular grid (left). Toroidal magnetic field distribution at the moment of its maximal energy for the initial quadrupole field (right).

into explosion energy takes place by means of the magnetic field [ 6$]$. Neutron stars are rotating, having magnetic fields up to $10^{13} \mathrm{Gs}$, and even more. In differentially rotating new born neutron stars radial magnetic field is twisted, and magnetic pressure becomes very high, producing MHD shock by which the rotational energy is transformed to the explosion energy.

Calculations of MRE have been done in [[13], using one-dimensional MHD equations, for the case of cylindrical symmetry. The calculations show that the main part of the envelope joins the neutron star, and the outer part of the envelope expands with large velocity, carrying out a considerable part of rotational energy and rotational momentum. MRE has an efficiency about $10 \%$ of the rotational energy, the ejected mass is $\approx 0.1$ of the star mass, explosion energy $\approx 10^{51}$ erg. Ejected mass and explosion energy depend weekly on the parameter $\alpha=E_{\text {mag }} / E_{\text {grav }}$ at initial moment. Explosion time depends on $\alpha$ as $t_{\text {expl }} \sim \frac{1}{\sqrt{\alpha}}$. Small $\alpha$ is difficult for numerical calculations with explicit numerical schemes because of the Courant restriction on the time step. At small $\alpha$ the hydrodynamic time scale is much smaller than the time of the magnetic field growth until explosion. Therefore at small $\alpha$ we have a "stiff" system of equations with two very different time scales.

\section{2-D calculations}

The numerical method used in simulations is based on the implicit operator-difference, completely conservative scheme on a Lagrangian triangular grid of variable structure, with grid reconstruction (Fig.W). The implicitness of the applied numerical scheme allows for large time-steps. It is important to use the implicit scheme in the presence of two strongly different time-scales: the small one due to huge sound velocity in the central parts of the star, and the big one determining the evolution of the magnetic field. The method applied here was developed and its stability was investigated in the papers of [团], [5], [四]. The scheme is fully conservative, what includes conservation of mass, momentum and total energy, and correct transitions between different types of energies. It was tested thoroughly with different tests by []].

In the calculations of magnetorotational core-collapse supernova performed by [B], MHD equations with self-gravitation, and infinite conductivity have been solved using the numerical scheme as described above. The problem has an axial symmetry $\left(\frac{\partial}{\partial \phi}=0\right)$, and the symmetry to the equatorial plane $(\mathrm{z}=0)$. Initial toroidal current $J_{\phi}$ was taken at the initial moment (time 

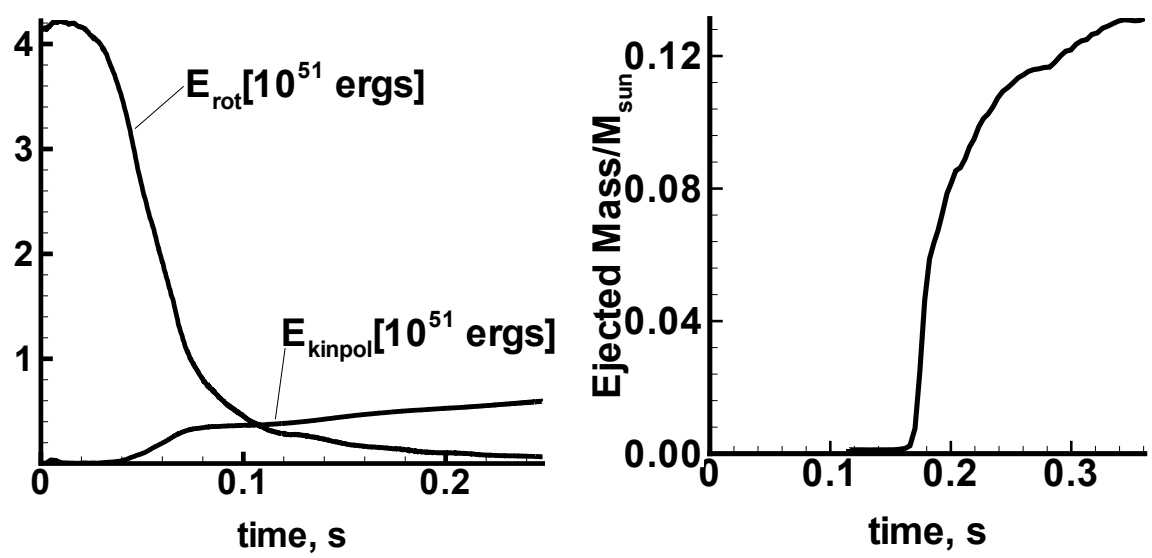

Figure 2: Time dependence of rotational, kinetic poloidal, and magnetic energies during explosion for a dipole -like field, from [24] (left). Time dependence of the ejected mass during the magnetorotational explosion with initial dipole magnetic field, from [24] (right).

started now from the stationary rotating neutron star) producing $H_{r}, H_{z}$ according to Biot-Savart law $\mathbf{B}=\frac{1}{c} \int_{V} \frac{\mathbf{J} \times \mathbf{R}}{R^{3}} d V$. Initial magnetic field of quadrupole-like symmetry is obtained at opposite directions of the current in both hemispheres. Neutrino cooling was calculated using a variant of a flux-limited method, [B]].

Magnetic field is amplified due to twisting by the differential rotation, and subsequent development of the magnetorotational instability. The field distribution for initial quadrupole-like magnetic field with $\alpha=10^{-6}$, at the moment of the maximal energy of the toroidal magnetic field is represented in Fig.W. The maximal value of $B_{\phi}=2.5 \cdot 10^{16}$ Gs was obtained in the calculations. The magnetic field at the surface of the neutron star after the explosion is $B=4 \cdot 10^{12}$ Gs. Time dependence during the explosion of rotational, gravitational, internal, and kinetic poloidal energies is given in Figs.2. Almost all gravitational energy,released during the collapse, is carried away by neutrino. The total energy ejected in the kinetic form is $\sim 0.6 \cdot 10^{51} \mathrm{erg}$, and the total ejected mass is equal to $\sim 0.14 M_{\odot}$.

The simulations were done for the initial poloidal magnetic field of quadrupole [B] and of dipole [24] types of symmetry. The initial ratios between the rotational and gravitational, and also between the internal and gravitational energies of the star had been chosen as: $\frac{E_{\text {rot }}}{E_{\text {grav }}}=0.0057$, $\frac{E_{\text {int }}}{E_{\text {grav }}}=0.727$. The initial magnetic field was "turned on" after the collapse stage. The ratio between the initial magnetic and gravitational energies was chosen as $10^{-6}$. The initial poloidal magnetic field in the center, at start of the evolution of the toroidal field was $\sim 3.2 \times 10^{13} \mathrm{G}$.

The magnetic field works as a piston for the originated MHD shock. The time dependence of the ejected mass and energy is given in Figs. [1] [3]. During the magnetorotational explosion $\sim 0.14 M_{\odot}$ of the mass and $\sim 0.6 \cdot 10^{51} \mathrm{ergs}(\sim 10 \%$ of the rotational energy) are ejected. The simulation of the MR supernova explosion for various initial core masses and rotational energies was done by [ए]]. The initial core mass was varied from $1.2 M_{\odot}$ to $1.7 M_{\odot}$, the initial specific rotational energy $E_{\text {rot }} / M_{\text {core }}$, was varied from $0.19 \times 10^{19}$ to $0.4 \times 10^{19} \mathrm{erg} / \mathrm{g}$. The explosive energy 

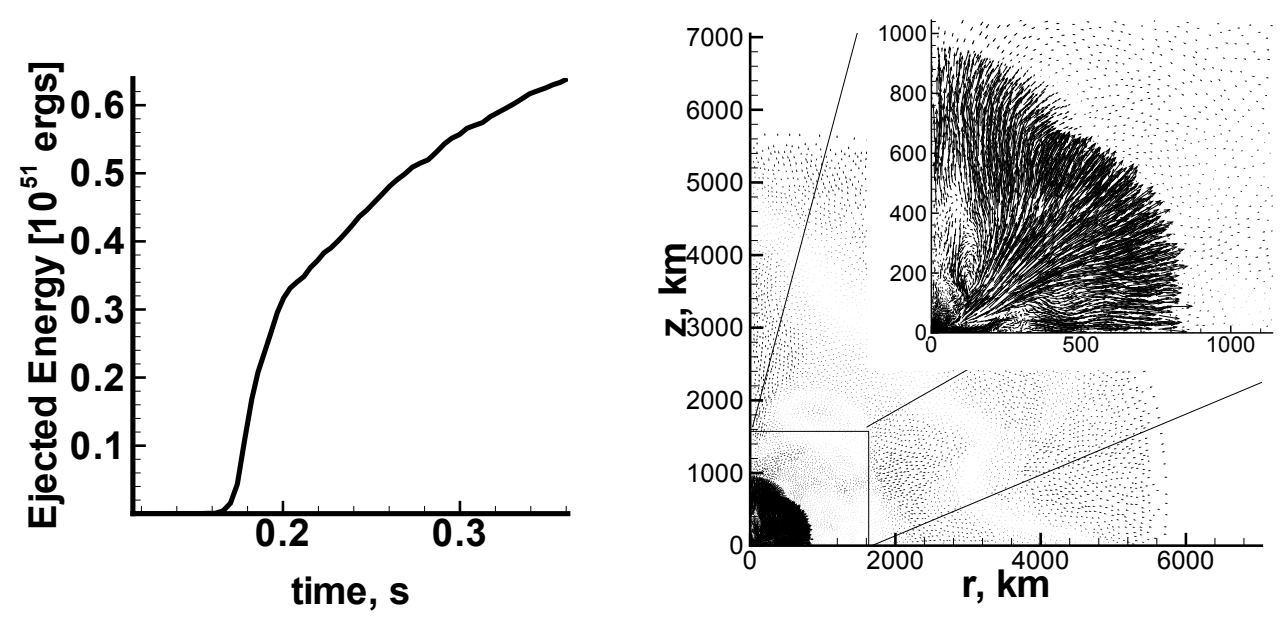

Figure 3: Time dependence of the ejected energy during the magnetorotational explosion with initial dipole magnetic field, from [24] (left). Time evolution of the velocity field (outflow) for the time moment $t=$ $0.075 \mathrm{~s}$, from [24] (right).

increases with the mass of the core, and the initial rotational energy. The energy released in MR explosion, $(0.5-2.6) \times 10^{51} \mathrm{erg}$, is sufficient to explain supernova with collapsing cores, Types II and Ib. The energies of Type Ic supernovae could be higher.

\section{Magnetorotational instability}

Magnetorotational instability (MRI) leads to exponential growth of magnetic fields. Different types of MRI have been studied by [B2], [28]. MRI starts to develop when the ratio of the toroidal to poloidal magnetic energies is becoming large. In 1-D calculations MRI is absent because of a restricted degree of freedom, and time of MR explosion is increasing with $\alpha$ as $t_{\text {expl }} \sim \frac{1}{\sqrt{\alpha}}$, $\alpha=\frac{E_{\text {mag } 0}}{E_{\text {grav }}}$. Due to development of MRI the time of MR explosion depends on $\alpha$ much weaker. The MR explosion happens when the magnetic energy is becoming comparable to the internal energy, at least in some parts of the star. While the starting magnetic energy linearly depends on $\alpha$, and MRI leads to exponential growth of the magnetic energy, the total time of MRE in 2-D is growing logarithmically with decreasing of $\alpha, t_{\text {expl }} \sim-\log \alpha$. These dependencies are seen clearly from 1$\mathrm{D}$ [[3]] and 2-D calculations [B] , [D4] giving the following explosion times $t_{\text {expl }}$ (in arbitrary units): $\alpha=0.01, t_{\text {expl }}=10, \quad \alpha=10^{-12}, t_{\text {expl }}=10^{6}$ in $1-\mathrm{D}$, and $\alpha=10^{-6}, t_{\text {expl }} \sim 6, \quad \alpha=10^{-12}, t_{\text {expl }} \sim$ 12 in 2-D. The dependence of the explosion time is shown in graphs for the quadrupole [[23], and dipole [24] configurations of the magnetic field.

\section{Jet formation in MRE}

Jet formation in MRE happens at the initial magnetic field of a dipole-like structure, when the ejected mass is collimated along the rotational axis [24], see Figs.3].].

Simulations of the MR supernova have been made with equation of state suggested in [D6]. A comparison of our results for the initially uniform magnetic field, with the results in [22] and 

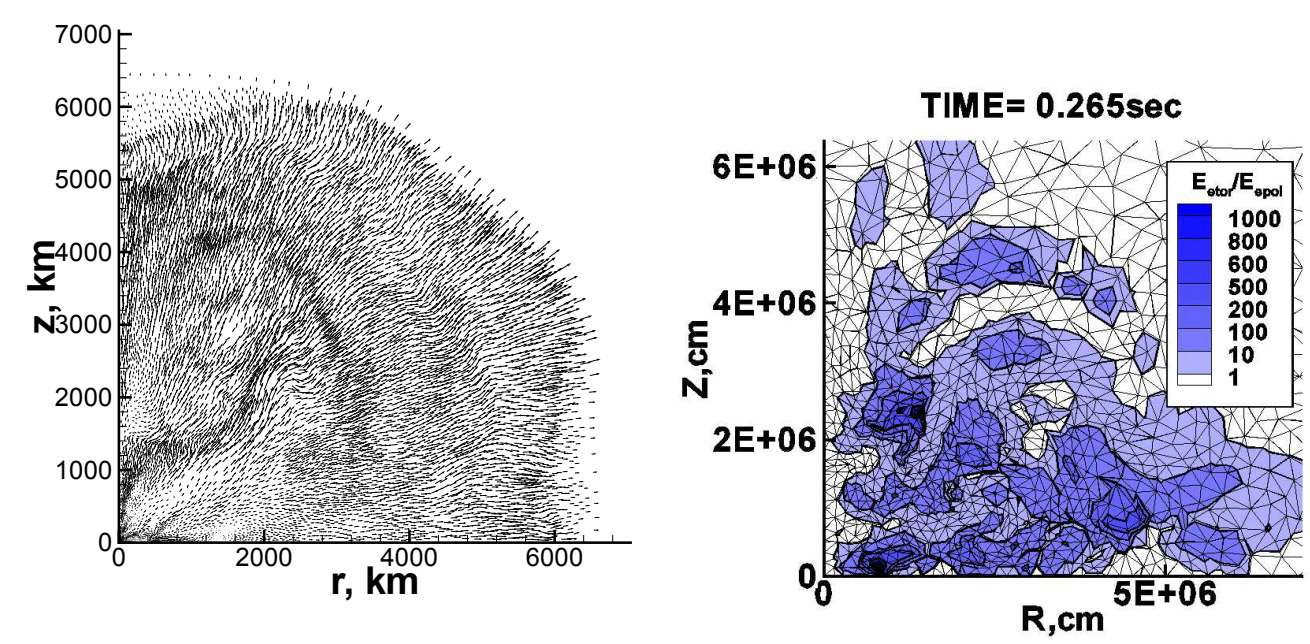

Figure 4: Time evolution of the velocity field (outflow) for the time moment, $t=0.25 s$, from [24] (left). The Lagrangian triangular grid and the ratio of the toroidal magnetic energy $E_{t o r}$ to the poloidal magnetic energy $E_{p o l}, \frac{E_{t o r}}{E_{p o l}}$ at $t=265 \mathrm{~ms}$ for the case $H_{0}=10^{9} \mathrm{G}, E_{\text {rot } 0} / E_{\text {grav } 0}=1 \%$ (right).

[BO], for the same initial and boundary conditions, shows good agreement for a strong initial field $\left(H_{0}=10^{12} \mathrm{G}\right)$, while for a weaker field $\left(H_{0}=10^{9} \mathrm{G}\right)$ we get mildly collimated jet-like explosion; see also [44]. MRI is developed in the case of a weaker initial magnetic field, and it is not present in the calculations with stronger field, see Figs.], 1 .

We have made simulations for the initial magnetic fields $H_{0}=10^{9}, 10^{12} \mathrm{G}$. The ratio of the initial rotational energy to the absolute value of the gravitational energy was taken $E_{\text {rot } 0} / E_{\text {grav } 0}=$ $1 \%, 2 \%$. When the initial magnetic field is moderate $\left(H_{0}=10^{9} \mathrm{G}\right)$ and $E_{\text {rot } 0} / E_{\text {grav } 0}=1 \%$ MDRI develops what means exponential growth of all components of the magnetic field (Fig.(G)).

At Fig. 19 The Lagrangian triangular grid and the ratio of the toroidal magnetic energy to the poloidal one $\left(E_{t o r} / E_{\text {pol }}\right)$ is represented for the case $H_{0}=10^{9} \mathrm{G}$, and $E_{\text {rot } 0} / E_{\text {grav } 0}=1 \%$. The toroidal magnetic energy dominates over the poloidal one in the significant part of the region where new neutron star is forming. The MDRI is well-resolved on our triangular grid.

In the case with $H_{0}=10^{12} \mathrm{G}$, and $E_{\text {rot } 0} / E_{\text {grav } 0}=1 \%$ there is no regions of domination of $E_{t o r}$ over $E_{p o l}$.

The Fig. $[$ represents a time evolution of rotational, magnetic poloidal and toroidal energies for MR explosion when $H_{0}=10^{9} \mathrm{G}$.

The Fig.6 is the same data plot as the Fig.5 but zoomed and the vertical axis is in logarithmic scale. The straight dash-dotted line at the Fig.6 shows the exponential growth of the toroidal and poloidal magnetic energies with the time due to the MDRI.

The rotational energy has two maxima. The first contraction is accompanied by the strong growth of the rotational energy due to angular momentum conservation, maximum of which coincides with the first maximum of the density. The first contraction, and the subsequent bounce, happens when the magnetic field is growing slowly, and the angular momentum losses from the stellar core are small. Development of the magnetorotational instability leads to a rapid growth of the magnetic field, large angular momentum flux from the core, what stops the expansion, and 
leads to the second contraction phase. In this case the contraction is not transforms into expansion, because of the rapid decrease of the rotational energy due to strong angular momentum flux outside from the core.

The energy of the poloidal magnetic field grows due to the contraction until the time $t \approx 0.225$ sec. Then it slightly decreases because of the formation of the bounce shock, and its motion outwards. The toroidal magnetic energy grows as quadratic function because of wrapping of the magnetic force lines (toroidal component of the magnetic field grows linearly). Starting from $t \approx 0.3 \mathrm{sec}$ both the poloidal and toroidal magnetic energies begin to grow exponentially due to MDRI. At $t \approx 0.36 \mathrm{sec}$ both magnetic energies comes to saturation. The MHD shock wave develops what leads to the MR explosion. The MR explosion develops in all directions without formation of a collimated flow.

The MR explosion for an extremely high initial magnetic field $\left(H_{0}=10^{12} \mathrm{G}\right)$ is developing in a qualitatively different way. The initial magnetic field is so strong that it grows strongly during the first contraction, and the explosion happens before the development of MDRI happens (Fig.D). At the Fig. $\square$ the time evolution of the rotational, poloidal magnetic and toroidal magnetic energies are represented. The rotational energy has one extremum at $t \approx 0.32 \mathrm{sec}$ corresponds to the maximal contraction, accompanied by a corresponding growth of of the toroidal and poloidal magnetic energies. The poloidal magnetic field grows due to the contraction, the toroidal magnetic field appears due to the differential rotation and is amplified both due to the differential rotation and the contraction of the core.

The strong initial magnetic field leads to a rapid loss of the angular momentum from the core already during the contraction phase. The centrifugal force becomes unimportant, and the first contraction is not followed by any bounce, leaving behind a dense slowly rotating neutron star core. We have got here a prompt explosion.

The force lines of the magnetic field play the role of 'rails'. The matter moves along the force lines. The magnetic pressure dominates a the periphery of the core. The MR explosion develops mainly along the axis of rotation, and the collimated flow is formed. The MR explosion results in the collimated jet. The degree of jet collimation is approximately the same as in [ख2]. For the case when $E_{\text {rot } 0} / E_{\text {grav } 0}=1 \%$ and $H_{0}=10^{9} \mathrm{G}$ the MR supernova explosion energy is $\sim 4 \times 10^{50}$ erg, for the $H_{0}=10^{12} \mathrm{G}$ the MR supernova explosion energy reaches the value of $\sim 8 \times 10^{50} \mathrm{erg}$. The explosion energy resulted in the simulations by Lagrangian method are close to those ones found in the simulations made by Eulerian scheme [2R] (excluding the case of $B_{0}=10^{12} G$ and $\left.E_{\text {rot } 0} /\left|E_{\text {grav } 0}\right|=1 / \%\right)$.

\section{Asymmetry of the explosion}

It follows from our simulations that MR supernova explosion arises after development of the MRI. The development of the MRI is a stochastic process and hence the resulting shape of the supernova can vary. It is important to point out that MR mechanism of supernova explosion leads always to asymmetrical outbursts.

In our simulations we suggested the symmetry to the equatorial plane. In reality this symmetry can be violated due to the MRI, simultaneous presence of the initial dipole and quadrupole -like magnetic field ([B4] $)$ and initial toroidal magnetic field ([Q⿴囗十). The violation of the symmetry could 


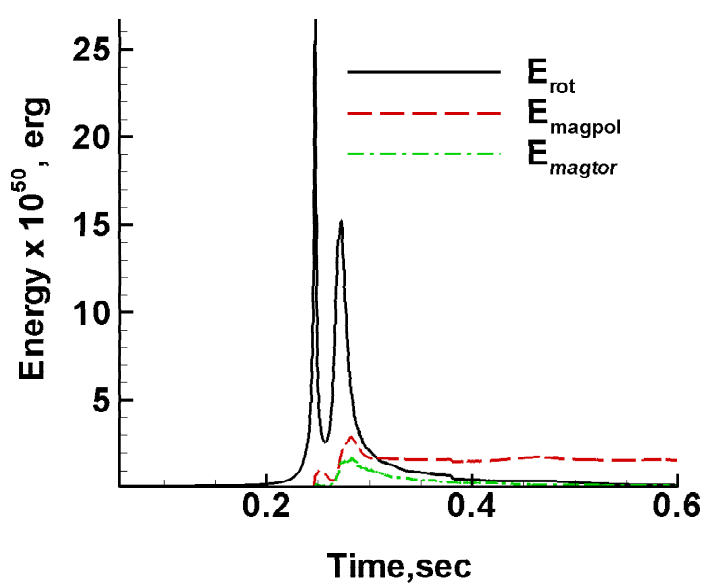

Figure 5: Time evolution of rotational energy $E_{\text {rot }}$ (solid line), magnetic poloidal energy $E_{\text {magpol }}$ (dashed line) and magnetic toroidal energy $E_{\text {magtor }}$ (dash-dotted line) for the case $H_{0}=10^{9} \mathrm{G}, E_{\text {rot } 0} / E_{\text {grav } 0}=1 \%$.
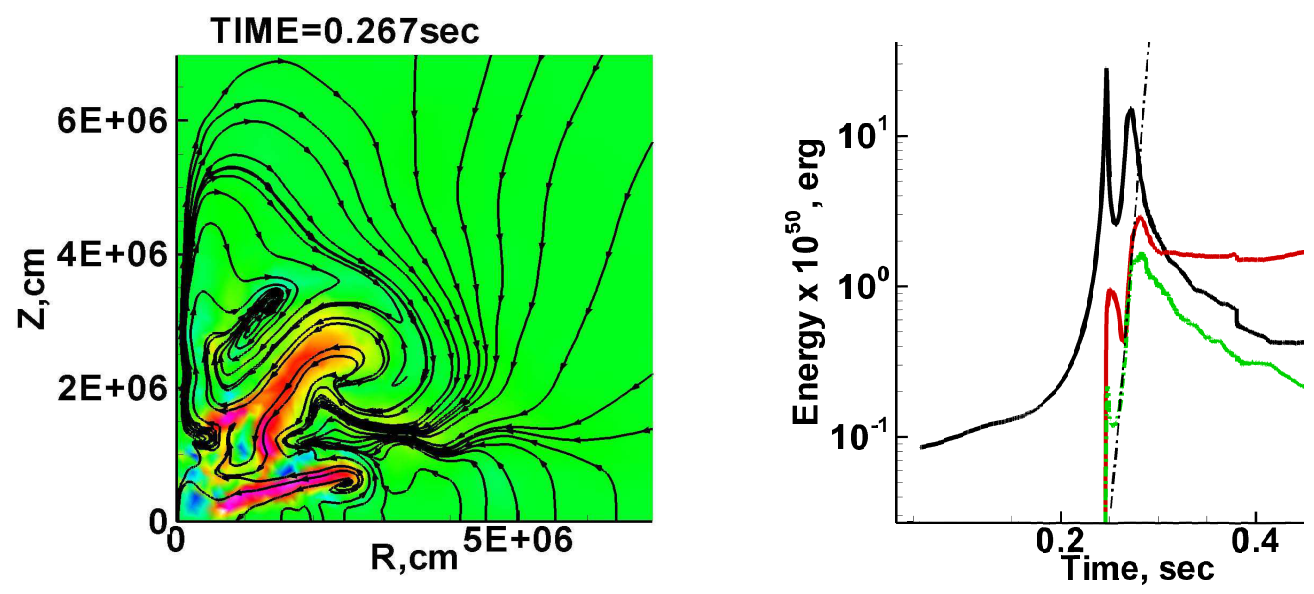

Figure 6: Developed MDRI due to convection and MRI/Tayler instability at $t=267 \mathrm{~ms}$ for the case $H_{0}=10^{9} \mathrm{G}, E_{\text {rot } 0} / E_{\text {grav } 0}=1 \%$ (contour plot - the toroidal magnetic field, arrow lines - force lines of the poloidal magnetic field) (left). Zoomed time evolution of rotational energy $E_{r o t}$ (solid line), magnetic poloidal energy $E_{\text {magpol }}$ (dashed line) and magnetic toroidal energy $E_{\text {magtor }}$ (dash-dotted line) for the case $H_{0}=10^{9} \mathrm{G}, E_{\text {rot } 0} / E_{\text {grav } 0}=1 \%$. Straight dash-dotted line shows exponential growth of the toroidal and poloidal magnetic energies (right). 

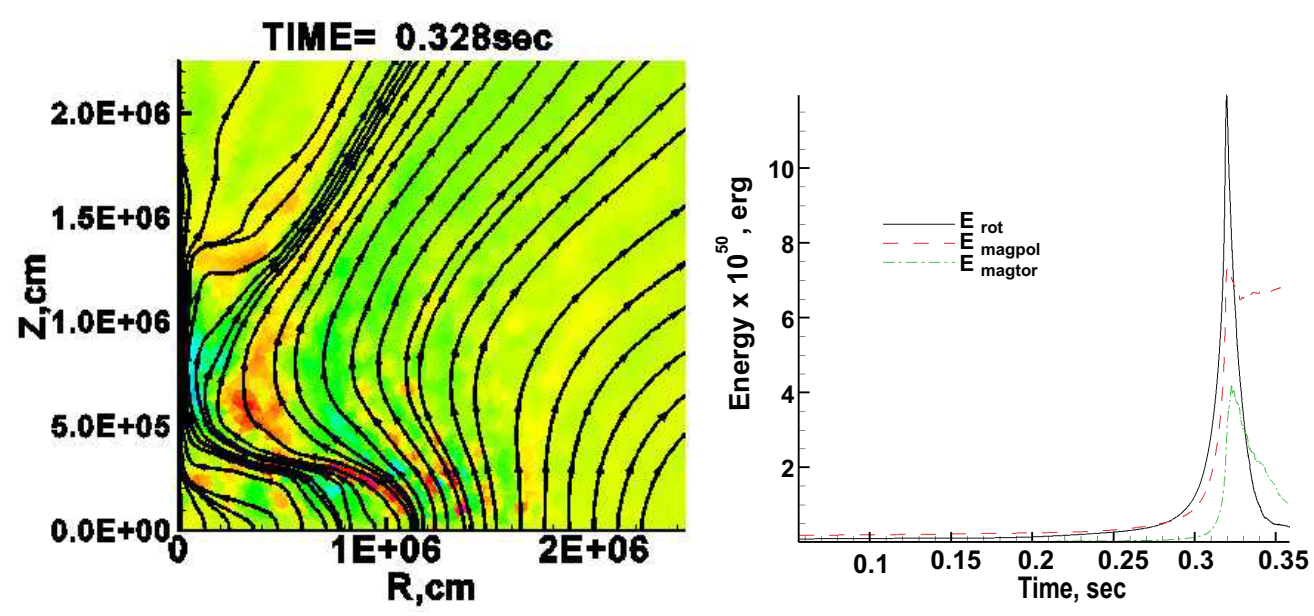

Figure 7: Absence of MDRI at $t=328 \mathrm{~ms}$ for the case $H_{0}=10^{12} \mathrm{G}, E_{\text {rot } 0} / E_{\text {grav } 0}=1 \%$ (contour plot the toroidal magnetic field, arrow lines - force lines of the poloidal magnetic field) (left). Time evolution of rotational energy $E_{r o t}$ (solid line), magnetic poloidal energy $E_{\text {magpol }}$ (dashed line) and magnetic toroidal energy $E_{\text {magtor }}$ (dash-dotted line) for the case $H_{0}=10^{12} \mathrm{G}, E_{\text {rot } 0} / E_{\text {grav } 0}=1 \%$ (right).

lead to the kick effect and formation of rapidly moving radio pulsars. A kick velocity, along the rotational axis, formed due to magnetohydrodynamic processes in presence of the asymmetry of the magnetic field, by estimations [ [ $]$ ], does not exceed $300 \mathrm{~km} / \mathrm{sec}$.

When rotational and magnetic axes do not coincide the whole picture of the explosion process is three dimensional. Nevertheless, the magnetic field twisting happens always around the rotational axis, so we may expect the kick velocity of the neutron star be strongly correlated with its spin direction. During the phase of MRE explosion the regular component of magnetic field may exceed temporally $10^{16} \mathrm{G}$ [B] , [24], when the neutrino cross-section depends on the magnetic field strength. The level of the anisotropy of the magnetic field relative to the plain perpendicular to the rotational axis [22] may be of the order of 50\%, leading to strong anisotropy of the neutrino flux. The kick velocity due to the anisotropy of the neutrino flux may reach several thousands $\mathrm{km} / \mathrm{c}[\mathrm{B}]$, explaining appearance of the most rapidly moving radio pulsars [33]. Simultaneously, because of the stochastic nature of MRI, the level of the anisotropy should be strongly variable, leading to a large spreading in the the neutron star velocities. This prediction of MR explosion differs from the models with a powerful neutrino convection, where arbitrary direction of the kick velocity is expected ([ㅍ],[미]).

Analysis of observations of pulsars shows that rotation and velocity vectors of pulsars are aligned, as is predicted by the MR supernova mechanism. This alignment was first found in [27], and was confirmed in the papers [ [20],[[2], [[25]. The alignment of the vectors can be violated in the case when the supernova explodes in a binary system.

\section{Conclusions}

In MRE the efficiency of transformation of rotational energy into the energy of explosion is $\sim 10 \%$. MRI strongly accelerates MRE, at lower values of the initial magnetic fields. Jet formation 
is possible for dipole-like topology of the field. MRE energy is not sensitive to the details of the equation of state, model of the neutrino transfer, and to the choice of the numerical scheme. The observed alignment of the rotation and velocity vectors of pulsars follows directly from the MRE supernova model.

\section{Acknowledgments}

The work of SGM and GSBK was supported partially by RFBR grants 14-02-00728 and 1429-06045, and by President RF grant NSh-261.2014.2.

\section{References}

[1] N.V. Ardeljan, G.S. Bisnovatyi-Kogan, K.V. Kosmachevskii, \& S.G. Moiseenko, An implicit Lagrangian code for the treatment of nonstationary problems in rotating astrophysical bodies, Astron. Ap. Suppl. 115 (1996) 573.

[2] N.V. Ardeljan, G.S. Bisnovatyi-Kogan, \& S.G. Moiseenko, Nonstationary magnetorotational processes in a rotating magnetized cloud, Astron. Ap. 355 (2000) 1181.

[3] N.V. Ardeljan, G.S. Bisnovatyi-Kogan, \& S.G. Moiseenko, Magnetorotational supernovae, MNRAS 359 (2005) 333.

[4] N.V. Ardelyan, \& S.V. Chernigovskii, About convergence of difference schemes for two dimensional gas dynamics equations in acoustic approximation, Diff. Uravneniya 20 (1984) 1119.

[5] N.V. Ardeljan, \& K.V. Kosmachevskii, Implicit free-Lagrange method for computing two-dimensional magnetogas-dynamic, Comput. Math. Model. 6 (1995) 209

[6] G.S. Bisnovatyi-Kogan, The Explosion of a Rotating Star As a Supernova Mechanism, Astron. Zh. 47 (1970) 813 [SvA 14 (1971) 652].

[7] G.S. Bisnovatyi-Kogan, Stellar Physics 2: Stellar Evolution and Stability, Astronomy and Astrophysics Library, Springer-Verlag Berlin Heidelberg 2011.

[8] G.S. Bisnovatyi-Kogan, Asymmetric neutrino emission and formation of rapidly moving pulsars, Astron. Ap. Transact. 3 (1993) 287.

[9] G.S. Bisnovatyi-Kogan, \& S.G. Moiseenko, Violation of the mirror symmetry of the magnetic field in rotating stars and possible astrophysical manifestations, Astron. Zh. 69 (1992) 563 [Soviet Astronomy 36 (1992) 285].

[10] G.S. Bisnovatyi-Kogan, S.G. Moiseenko, \& N.V. Ardelyan, Different magneto-rotational supernovae, Astronomy Reports 52 (2008) 997.

[11] G.S. Bisnovatyi-Kogan, S.G. Moiseenko, \& N.V. Ardelyan, Magnetorotational Explosions of Core-Collapse Supernovae, Acta Polytechnica CTU Proceedings 1(1) (2014) 181 [astro-ph/1408.2395].

[12] G.S. Bisnovatyi-Kogan, S.G. Moiseenko, \& N.V. Ardeljan, Magnetorotational Explosions of Core-collapse Supernovae, ASP Conference Series 474 (2013) 47.

[13] G.S. Bisnovatyi-Kogan, Yu.P. Popov, \& A.A. Samokhin, The magnetohydrodynamic rotational model of supernova explosion, Ap. Space Sci 41 (1976) 287. 
[14] A. Burrows, L. Dessart, E. Livne, C.D. Ott, \& J. Murphy, Simulations of Magnetically Driven Supernova and Hypernova Explosions in the Context of Rapid Rotation, ApJ 664 (2007) 416.

[15] A. Burrows, J. Hayes, \& B.A. Fryxell, On the Nature of Core-Collapse Supernova Explosions, ApJ 450 (1995) 830.

[16] S.A. Colgate, \& R.H. White, The Hydrodynamic Behavior of Supernovae Explosions, ApJ 143 (1966) 626.

[17] L. Dessart, A. Burrows, E. Livne, \& C.D. Ott, Multidimensional Radiation/Hydrodynamic Simulations of Proto-Neutron Star Convection, ApJ 645 (2006) 534.

[18] R. Epstein, Lepton-driven convection in supernovae, MNRAS 188 (1979) 305.

[19] H.-T. Janka, \& E. Müller, The First Second of a Type II Supernova: Convection, Accretion, and Shock Propagation, ApJ 448 (1995) L109.

[20] S. Johnston, G. Hobbs, S. Vigeland, M. Kramer, J.M. Weisberg, \& A.G. Lyne, Evidence for alignment of the rotation and velocity vectors in pulsars, MNRAS 364 (2005) 1397.

[21] S. Johnston, M. Kramer, A. Karastergiou, G. Hobbs, S. Ord, \& J. Wallman, Evidence for alignment of the rotation and velocity vectors in pulsars - II. Further data and emission heights, MNRAS 381 (2007) 1625.

[22] H. Mikami, Y. Sato, T. Matsumoto, \& T. Hanawa, Three-dimensional Magnetohydrodynamical Simulations of a Core-Collapse Supernova, ApJ 683 (2008) 357.

[23] S.G. Moiseenko, G.S. Bisnovatyi-Kogan, \& N.V. Ardeljan, Magnetorotational Supernova Simulations, ASP Conference Series 342 (2005) 190.

[24] S.G. Moiseenko, G.S. Bisnovatyi-Kogan, \& N.V. Ardeljan, A magnetorotational core-collapse model with jets, MNRAS, 370 (2006) 501.

[25] A. Noutsos, M. Kramer, P. Carr, S. Johnston, Pulsar spin-velocity alignment: further results and discussion, MNRAS 423 (2012) 2736.

[26] H. Shen, H. Toki, K. Oyamatsu, \& K. Sumiyoshi, Relativistic equation of state of nuclear matter for supernova and neutron star, Nuclear Physics A 637 (1998) 435.

[27] T.V. Smirnova, V.I. Shishov, V.M. Malofeev, The Spatial Structure of Pulsar Emission Sources Determined Using Interstellar Scintillation, ApJ 462 (1996) 289.

[28] H.C. Spruit, Dynamo action by differential rotation in a stably stratified stellar interior, Astron. Ap. 381 (2002) 923.

[29] T. Takiwaki, K. Kotake, S. Nagataki, \& K. Sato, Magneto-driven Shock Waves in Core-Collapse Supernovae, ApJ 616 (2004) 1086

[30] T. Takiwaki, K. Kotake, \& K. Sato, Dynamo action by differential rotation in a stably stratified stellar interior, ApJ 691 (2009) 1360.

[31] R. Tayler, The adiabatic stability of stars containing magnetic fields-I.Toroidal fields, MNRAS 161 (1973) 365

[32] E.P. Velikhov, Stability of an ideally conducting liquid flowing between cylinders rotating in a magnetic field J. Exper. Theor. Phys. 36 (1959) 1398 [Sov. Phys. JETP 9 (1959) 995].

[33] W.H.T. Vlemmings, S. Chatterjee, W.F. Brisken, et al., Pulsar Astrometry at the Microarcsecond Level, MmSAI 76 (2005) 531 [astro-ph/0509025]. 
[34] J.C L. Wang, M.E. Sulkanen, \& R.V.E. Lovelace, Intrinsically asymmetric astrophysical jets, ApJ 390 (1992) 46. 